\title{
Prognostic Tests and Antiepileptic Drug Withdrawal After Epilepsy Surgery
}

\author{
Adil Harroud, Alexander G. Weil, Alain Bouthillier, Dang Khoa Nguyen
}

\begin{abstract}
Approximately one in three patients with a successful epilepsy surgery will have seizure recurrence following antiepileptic drugs (AED) withdrawal. The value of postoperative testing for predicting seizure relapse after AED tapering is not clear. The purpose of this study was to review the literature for evidence on the use of postoperative investigations before AED discontinuation after successful epilepsy surgery. We were unable to identify studies on the prognostic value of postoperative magnetic resonance imaging and AED blood levels. The literature review yielded seven studies on the predictive value of electroencephalography. Four studies found no association between interictal discharges (IED) and seizure relapse. These studies suffered from various limitations due to their retrospective design and generally small cohorts. Two of the three studies reporting a positive association were prospective and provided strong evidence of an increased risk of seizure recurrence with presence of postoperative IED in successfully operated patients undergoing AED withdrawal.
\end{abstract}

RÉSUMÉ: Tests pronostiques et arrêt de la médication antiépileptique après une chirurgie de l'épilepsie. Environ un patient sur trois qui subit avec succès une chirurgie aura une récidive suite au retrait de la médication antiépileptique (MAÉ). L'utilité des tests postopératoires pour prédire la récurrence des crises après le retrait progressif de la MAÉ n'est pas clairement établie. Le but de cette étude était de revoir la littérature afin de documenter l'utilisation d'examens postopératoires avant le retrait des MAÉ suite à une chirurgie de l'épilepsie couronnée de succès. Nous n'avons pas pu identifier d'études portant sur la valeur pronostique de l'imagerie par résonance magnétique postopératoire et les niveaux de MAÉ. La revue de littérature a identifié sept études sur la valeur prédictive de l'électroencéphalographie (ÉEG). Dans quatre études aucune association n'a été mise en évidence entre les décharges interictales (DII) et la récurrence des crises. Ces études comportaient différentes limites à cause de leur devis d'étude et du nombre limité de sujets. Deux des trois études rapportant une association positive étaient des études prospectives et présentaient des données mettant en évidence une augmentation du risque de récurrence des crises lors du retrait des MAÉ, lorsque des DII étaient présentes après la chirurgie chez des patients dont l'intervention avait été un succès.

Can J Neurol Sci. 2014; 41: 409-412

In patients with successful epilepsy surgery, the benefits of stopping antiepileptic drugs (AED) must be weighed against the risk of seizure relapse, which occurs in about one in three patients $^{1,2}$. While there have been many reports on AED discontinuation in medically treated patients ${ }^{3-6}$, evidence on seizure-free patients after epilepsy surgery is still scant. Some clinical and presurgical variables have been proposed to guide this decision. Postoperative tests have also been used in an attempt to identify patients at low risk of seizure recurrence before a trial of AED discontinuation, but their pertinence remains unclear. For example, some physicians may feel more comfortable with AED discontinuation in the presence of low levels of AED (from poor compliance) in a seizure-free postoperative patient, when magnetic resonance imaging (MRI) confirms that the intended area has indeed been resected and when a postoperative electroencephalography (EEG) fails to identify epileptiform discharges. Recent surveys of United States (US) and Canadian epileptologists have evaluated, among other factors, the frequency with which postoperative AED blood levels, MRI and EEG should be obtained before stopping $\mathrm{AED}^{7,8}$. In both studies, the majority of respondents reported obtaining an EEG, but only infrequently requested an MRI. The use of AED serum levels was more frequent in the US than in
Canadian centers. These studies highlighted the high variability in practices surrounding AED discontinuation. In the absence of clear guidelines, the purpose of this study was to review the literature for evidence on the use of postoperative investigations before AED discontinuation after successful epilepsy surgery.

\section{METHODS}

An electronic search of the Medline database was performed. The search strategy used the key words "epilepsy surgery," "antiepileptic drug" or "anticonvulsant," and "withdrawal" or "discontinuation." The search was limited to publications in English or French. The titles and abstracts of all search results

From the Division of Neurology (AH, DKN), Division of Neurosurgery (AGW, AB), Hôpital Notre-Dame du CHUM, Université de Montréal, Montreal, Quebec, Canada. Received October 10, 2013. Final Revisions Submitted January 8, 2014. Correspondence to: Dang K. Nguyen, Division of Neurology, Hôpital Notre-Dame du CHUM, 1560 rue Sherbrooke Est, Montreal, Quebec, H2L 4M1, Canada. Email: d.nguyen@umontreal.ca. 
were screened for potentially relevant studies. Pertinent studies from the reference lists of all identified articles were also considered for review. The following criteria were used to include studies: (a) patients who underwent epilepsy surgery, temporal or extratemporal; (b) attempted AED discontinuation; and (c) reported prognostic value of postoperative AED serum levels, MRI and/or EEG on seizure recurrence after AED tapering.

The search yielded 37 results, with an additional 13 retrieved from references. Of those studies, we identified 8 original articles investigating the prognostic value of postoperative EEG. One study by Rathore et $a l^{9}$ was later excluded because it presented data on the predictive value of postoperative EEG that were included in a more complete article by the same group ${ }^{10}$. Seven studies on the utility of postoperative EEG were thus included in this review. Regarding the use of postoperative MRI, we identified three studies addressing the predictive value of either extent of surgery ${ }^{11,12}$ or completeness of surgery ${ }^{13}$ in successfully operated patients undergoing AED discontinuation. However, none of these studies evaluated the extent of the resection with a postoperative MRI. No studies on the pertinence of AED serum levels were found in our review.

For each of the studies pertaining to postoperative EEG, we retrieved, whenever available, demographic data, the number of patients operated on and undergoing AED tapering, the time between surgery and AED discontinuation, as well as time to EEG, mean follow-up, and the recurrence rate for normal and abnormal postoperative EEG.

\section{ReSUlts}

\section{Seizure recurrence and $A E D$ serum levels in $A E D$ discontinuation}

There were no studies pertaining to the role of AED serum levels before attempting AED discontinuation in patients with a successful epilepsy surgery.

\section{Seizure recurrence and MRI in AED discontinuation}

No studies looked specifically at the prognostic value of postoperative MRI before attempting AED discontinuation. Although two studies ${ }^{11,12}$ analyzed the extent of anterior temporal resection in cases of temporal lobe epilepsy and found that it did not influence seizure recurrence following AED discontinuation, neither of these studies specified having controlled the extent of resection with a postoperative MRI. Similarly, another study by Park et $a l^{13}$ looked at the impact of completeness of resection on seizure outcome after AED tapering in cases of extratemporal lobe epilepsy. However, the completeness of resection was assessed based on preoperative MRI, EEG recordings and intraoperative findings. There was no mention of postoperative MRI to control the completeness of resection.

\section{Seizure recurrence and EEG in $A E D$ discontinuation}

A total of seven studies were retained for analysis of the use of postoperative EEG as a predictor of seizure recurrence before AED discontinuation (Table 1). The study by Lachhwani et al ${ }^{14}$ focused exclusively on pediatric patients, while the other studies mainly included adults. Three studies showed a significant positive association between the presence of interictal epileptiform discharges (IED) on postoperative EEG and the risk of seizure recurrence following AED discontinuation ${ }^{9,15,16}$ (Table 2). This was in contrast to the four other studies in which this association was not shown to be significant ${ }^{11,12,14,17}$. However, all the studies that found no relationship between postoperative EEG and seizure recurrence were retrospective, and some included a relatively limited number of patients (Table 1). Also, the timing of the EEG with regard to surgery was variable and not clearly specified. Moreover, for two of these studies the postoperative EEG was not performed systematically in all patients undergoing a trial of AED tapering. In one study, the relationship between postoperative EEG and seizure recurrence was assessed for only $39(51 \%)$ of the 77 patients who underwent a trial of AED tapering ${ }^{11}$. Similarly, in another article

Table 1: Characteristics of included studies about the predictive value of postoperative EEG in AED discontinuation after successful epilepsy surgery

\begin{tabular}{|c|c|c|c|c|c|c|c|c|}
\hline Study & Design & Epilepsy & $\begin{array}{l}\text { Age at } \\
\text { surgery } \\
\text { (years) }\end{array}$ & $\begin{array}{l}\text { Population } \\
\text { (N) }\end{array}$ & $\begin{array}{l}\text { AED tapering } \\
\text { and postop } \\
\text { EEG (N) }\end{array}$ & $\begin{array}{l}\text { Mean time to } \\
\text { tapering } \\
\text { (months) }\end{array}$ & $\begin{array}{l}\text { Mean FU } \\
\text { in years } \\
\text { (range) }\end{array}$ & $\begin{array}{l}\text { Time to EEG } \\
\text { (months) }\end{array}$ \\
\hline Menon et al. 2012 & Prospective & $\begin{array}{l}\text { ET }(98 \% \\
\text { lesional) }\end{array}$ & $19.7(3-45)$ & 106 & 94 & $\begin{array}{l}3(\text { if }>1 \text { AED) } \\
12(\text { if }=1 \\
\text { AED })\end{array}$ & $4.6(2-11)$ & $3,12,24,36$ \\
\hline Rathore et al. 2011 & Prospective & $\mathrm{T}$ & $27.4+9.2$ & $262,225 \mathrm{SF}$ & 225 & $\begin{array}{l}3(\text { if }>1 \text { AED }) \\
12(\text { if }=1 \\
\text { AED })\end{array}$ & $4.8(1-12)$ & $3,12,24,36$ \\
\hline Lachhwani et al. 2008 & Retrospective & $\mathrm{T}+\mathrm{ET}$ & $11(0.25-18)$ & $252,97 \mathrm{SF}$ & 68 & 13 & 2 & NS \\
\hline Jeha et al. 2006 & Retrospective & $\mathrm{T}$ & NS & $371,231 \mathrm{SF}$ & 77 & NS & $\mathrm{NS}$ & 6 \\
\hline Kim et al. 2005 & Retrospective & $\mathrm{T}$ & $27.3(11-41)$ & $88,66 \mathrm{SF}$ & 60 & NS & $\mathrm{NS}$ & NS \\
\hline Schiller et al. 2000 & Retrospective & $\mathrm{T}+\mathrm{ET}$ & $31.9(9-55)$ & $493,210 \mathrm{SF}$ & 84 & 12 & $>12$ & NS \\
\hline Murro et al. 1991 & Retrospective & $\mathrm{T}$ & $30 *(9-61)$ & 104 & 39 & 12 & NS & NS \\
\hline
\end{tabular}

*=median; NS=not specified; SR=seizure-free; FU=follow-up; T=temporal; ET=extratemporal; postop=postoperative 
Table 2: Prognostic significance of postoperative EEG and seizure recurrence rates

\begin{tabular}{lllll}
\hline Study & Recurrence with normal EEG & Recurrence with abnormal EEG & Odds ratio & p-value \\
\hline Menon et al. 2012* & $23 / 63(36.5 \%)$ & $21 / 31(67.7 \%)$ & 1.85 & 0.004 \\
Rathore et al. 2011* & $15 / 162(9.3 \% \%)$ & $12 / 56(21.4 \%)$ & 2.30 & 0.03 \\
Lachhwani et al. 2008 & NS & NS & NS & Not significant \\
Jeha et al. 2006 & NS & NS & 4.29 & $95 \%$ CI: $1.21-15.13$ \\
Kim et al. 2005 & NS & $8 / 32(25 \%)$ & NS & Not significant \\
Schiller et al. 2000 & 10/52 (19\%) & NS & 1.32 & 0.54 \\
Murro et al. 1991 & NS & NS & Not significant \\
\hline
\end{tabular}

*=data presented for 1-year postoperative EEG. NS=Not specified

the AED regimen was reduced in 180 patients, but postoperative EEG data were only available for a subgroup of 84 (46.7\%) patients $^{12}$. In the other two studies ${ }^{14,17}$, all patients with AED tapering had a postoperative EEG, but data pertaining to seizure recurrence rates in patients with normal and abnormal postoperative EEG were not shown in their paper, precluding further analysis.

The retrospective study by Jeha et $a l^{16}$ found that IED on six month postoperative EEG was the only variable correlating with seizure recurrence following attempted AED withdrawal, but again, data were not shown. The two other studies showing a positive association between postoperative EEG and seizure recurrence in the context of AED tapering were prospective. Both studies were performed by the same group and with a similar design while addressing two distinct populations. The study by Rathore et $a l^{9}$ focused on patients with mesial temporal lobe epilepsy who underwent anterior temporal lobectomy, while the study by Menon et $a l^{15}$ addressed patients with extemporal epilepsy, mainly lesional (104/106 patients). In both studies, AED tapering was attempted for all patients who were free from seizures (including auras) for a sufficient period of time ( 3 or 12 months after surgery if on poly- or monotherapy respectively, or two years of seizure-freedom if postoperative seizures were present). Also, all patients systematically had an EEG at 3, 12, 24 and 36 months after surgery. Findings on postoperative EEGs were not taken into account during the AED tapering, with all the patients following a rather systematic protocol of AED reduction.

In patients with mesial temporal lobe epilepsy ${ }^{10}$, after excluding patients who had seizure recurrence during the first year after surgery, the presence of IED on the one year postoperative EEG significantly increased the risk of seizure recurrence following attempted AED withdrawal by 2.3-fold $(p=0.03)$ (Table 2). Interestingly, a three month postoperative EEG was not predictive of seizure recurrence after AED withdrawal. Prognostic values of EEG at two years and three years were also evaluated, with a positive association being found only for the latter. However, as patients who had already experienced a seizure relapse were not retrieved from analysis at these time points, the presence of IED could be simply a consequence of poor seizure control rather than predictive of seizure recurrence during AED tapering. The authors reported a sensitivity and specificity for one year postoperative EEG of 0.21 and 0.90 respectively. However, these values were calculated without excluding the five patients who had already experienced seizure recurrence before one year. When these patients were accounted for, sensitivity was 0.44 and specificity 0.77 .

In the study of patients with extratemporal epilepsy ${ }^{15}$, postoperative EEGs performed at one week, three months and one year after surgery were reported. Of those, only one week and one year postoperative EEGs were predictive of seizure relapse $(\mathrm{OR}=2.1, \mathrm{p}=0.016$; and $\mathrm{OR}=2.4, \mathrm{p}=0.004$, respectively). No patients had a seizure recurrence in the first year, meaning that the presence of IED was not secondary to early seizure relapse.

\section{DISCUSSION}

While there is abundant literature on AED discontinuation in medically treated patients ${ }^{3-6}$, evidence guiding AED tapering in patients with successful epilepsy surgery is still limited. In particular, the utility of postoperative testing to predict the risk of seizure recurrence is unclear. Hence, practices are highly variable between centers (based on local customs), even within centers (depending on where each individuals were trained) or throughout the years (depending on available studies at the time $)^{7,8}$. For example, in a recent retrospective analysis of 275 consecutive patients who underwent epilepsy surgery at our center between 1986 and 2011, we found that only 49 (35\%) of the 141 patients who attempted discontinuation of AED had a postoperative EEG. Fifteen (45.4\%) of the 33 patients with a normal EEG had seizure recurrence, compared to 9 (56.2\%) of the 16 patients with IED. This difference did not reach statistical significance $(\mathrm{p}=0.478)$, most likely due to the retrospective design and low power of the study ${ }^{18}$.

In this literature review, we attempted to assemble all the current evidence regarding postoperative investigations before AED withdrawal in patients with successful epilepsy surgery. We were unable to identify any studies about AED serum levels before AED tapering, even though that test was more often than not requested by many neurologists in the US and Canada in recent surveys ${ }^{7,8}$. While two studies evaluated the impact of the extent of resection in temporal lobe epilepsy surgery on seizure recurrence after AED tapering, neither of these studies controlled the extent of resection with a postoperative MRI. No significant relationship was found between the extent of resection and seizure outcome. However, several studies have reported that the intended extent of resection or intraoperative measurements can be very imprecise compared to the achieved 
resection on postoperative MRI ${ }^{19}$. The same limitation applies to the study by Park et $a l^{13}$ in which the association between completeness of resection and seizure recurrence after AED discontinuation was found to be significant. Hence, based on this review, we cannot recommend for or against postoperative MRI before AED tapering.

In this review, seven studies addressed the utility of postoperative EEG in AED discontinuation. Four authors found no association between the presence of IED and the risk of seizure recurrence, while three others did find a positive association. Five studies were retrospective and subject to various limitations. Some of these studies included only a limited number of patients. Except for the study by Jeha et al, the timing of the postoperative EEG was variable and its temporal relationship to AED discontinuation and seizure recurrence was not specified. Also, only a variable subgroup of seizure-free patients attempted AED discontinuation, which could affect the internal validity of these studies. Additionally, in two of these studies only a proportion of patients who underwent AED withdrawal had a postoperative EEG, introducing a potentially important selection bias.

The studies by Rathore et $a l^{10}$ and Menon et $a l^{15}$ avoid many of these limitations. Data were collected prospectively and the groups were homogenous and relatively large in size. Also, the fact that all patients who were seizure-free for a sufficient period of time attempted AED discontinuation, along with the fact that all of them had postoperative EEGs, strengthens the internal validity of the study. There was an increased risk of seizure recurrence after AED tapering in patients with IED on 1-year postoperative EEG in both patients with mesial temporal and extratemporal epilepsy surgery. The three month EEG was not predictive of seizure recurrence. It could be that proximity to surgery renders it more susceptible to postoperative modifications and cortical irritation, reducing its predictive value. Data on two year and three year EEG in patients with mesial temporal lobe epilepsy were hardly interpretable, as patients with seizure recurrence before that time were not excluded from the calculation of its predictive value. Only mesial temporal lobe epilepsy and lesional extratemporal epilepsy were addressed by these two studies. Although IED on one year postoperative EEG seem to increase the rate of seizure relapse in this population, a significant proportion of patients with IED still remained seizure-free after discontinuation of AED. Hence, an abnormal postoperative EEG is not an absolute contraindication to attempting AED tapering, but should be considered together with other predictors to guide the joint decision made by physician and patient. Although a randomized clinical trial would be helpful in confirming these findings (and address other areas of uncertainty surrounding AED discontinuation after epilepsy surgery such as delay before tapering), it is unclear if such an ominous task is feasible. Among the many hurdles are patient heterogeneity, variety in surgical procedures, patient preference, high number of patients required, necessity for a long duration study to adequately assess recurrence, and convincing epilepsy specialists in different centers with a variety of biases and local customs to participate.

\section{CONCLUSION}

There is currently no evidence regarding the utility of postoperative AED serum levels or MRI in predicting the risk of seizure recurrence following AED tapering. Presence of IED on postoperative EEG one year after surgery seems to increase the risk of seizure recurrence in patients with both successfully operated mesial temporal lobe epilepsy and lesional extratemporal epilepsy.

\section{REFERENCES}

1. Schmidt D, Baumgartner C, Loscher W. Seizure recurrence after planned discontinuation of antiepileptic drugs in seizure-free patients after epilepsy surgery: a review of current clinical experience. Epilepsia. 2004;45(2):179-86.

2. Berg AT, Vickrey BG, Langfitt JT, et al. Reduction of AEDs in postsurgical patients who attain remission. Epilepsia. 2006;47 (1):64-71.

3. Shih JJ, Ochoa JG. A systematic review of antiepileptic drug initiation and withdrawal. Neurologist. 2009;15(3):122-31.

4. Wilkins DE. A guideline for discontinuing antiepileptic drugs in seizure-free patients. Neurology. 1999;53(1):239.

5. Shinnar S, Vining EP, Mellits ED, et al. Discontinuing antiepileptic medication in children with epilepsy after two years without seizures. A prospective study. N Engl J Med. 1985;313(16): 976-80.

6. Lossius MI, Hessen E, Mowinckel P, et al. Consequences of antiepileptic drug withdrawal: a randomized, double-blind study (Akershus Study). Epilepsia. 2008;49(3):455-63.

7. Tellez-Zenteno JF, Ronquillo LH, Jette N, et al. Discontinuation of antiepileptic drugs after successful epilepsy surgery. a Canadian survey. Epilepsy Res. 2012;102(1-2):23-33.

8. Berg AT, Langfitt JT, Spencer SS, Vickrey BG. Stopping antiepileptic drugs after epilepsy surgery: a survey of U.S. epilepsy center neurologists. Epilepsy Behav. 2007;10(2): 219-22.

9. Rathore C, Panda S, Sarma PS, Radhakrishnan K. How safe is it to withdraw antiepileptic drugs following successful surgery for mesial temporal lobe epilepsy? Epilepsia. 2011;52(3):627-35.

10. Rathore C, Sarma SP, Radhakrishnan K. Prognostic importance of serial postoperative EEGs after anterior temporal lobectomy. Neurology. 2011;76(22):1925-31.

11. Murro AM, King DW, Smith JR, et al. Withdrawal of antiepileptic medications following epilepsy surgery. J Epilepsy. 1991;4(1): $39-42$.

12. Schiller Y, Cascino GD, So EL, Marsh WR. Discontinuation of antiepileptic drugs after successful epilepsy surgery. Neurology. 2000;54(2):346-9.

13. Park K-I, Lee SK, Chu K, et al. Withdrawal of antiepileptic drugs after neocortical epilepsy surgery. Ann Neurol. 2010;67(2): 230-8.

14. Lachhwani DK, Loddenkemper $\mathrm{T}$, Holland $\mathrm{KD}$, et al. Discontinuation of medications after successful epilepsy surgery in children. Pediatr Neurol. 2008;38(5):340-4.

15. Menon R, Rathore C, Sarma SP, Radhakrishnan K. Feasibility of antiepileptic drug withdrawal following extratemporal resective epilepsy surgery. Neurology. 2012;79(8):770-6.

16. Jeha LE, Najm IM, Bingaman WE, et al. Predictors of outcome after temporal lobectomy for the treatment of intractable epilepsy. Neurology. 2006;66(12):1938-40.

17. Kim YD, Heo K, Park SC, et al. Antiepileptic drug withdrawal after successful surgery for intractable temporal lobe epilepsy. Epilepsia. 2005;46(2):251-7.

18. Harroud A, Weil AG, Bouthillier A, Nguyen DK. Antiepileptic drugs withdrawal after successful epilepsy surgery: utility of postoperative EEG [abstract]. Epilepsia. 2013;54(Suppl. 3):69.

19. Schramm J. Temporal lobe epilepsy surgery and the quest for optimal extent of resection: a review. Epilepsia. 2008;49(8): 1296-307. 